\title{
Test of the Hypothesis of Realism Using a Modified Version of Wigner Inequality
}

\author{
Nikolay Nikitin ${ }^{1,2,3, *}$ and Konstantin Toms ${ }^{4, * *}$ \\ ${ }^{1}$ Lomonosov Moscow State University, Department of Physics, Russia \\ ${ }^{2}$ Lomonosov Moscow State University, Skobeltsyn Institute of Nuclear Physics, Russia \\ ${ }^{3}$ Institute for Theoretical and Experimental Physics, Russia \\ ${ }^{4}$ Department of Physics and Astronomy, University of New Mexico, USA
}

\begin{abstract}
In the current work we present a modified Wigner inequality for test of the hypothesis of realism. This modified Wigner inequality is suitable for test of realism not only in systems that obey the laws of non-relativistic quantum mechanics, but also in systems that obey the laws of quantum field theory. We show how this inequality can be violated in systems of neutral pseudoscalar mesons.
\end{abstract}

\section{Introduction}

Already in the first years of quantum theory development one of the key questions was posed that resulted in creation of a new field: foundations of quantum mechanics. This question may be formulated as follows: "How the paradigm of quantum physics distincs from the paradigm of classical physics?". We consider special and general relativity theories as a part of classical physics. Final answer to this question is still not obtained, however the understanding of this question has advanced quite far comparing to the famous discussions of the beginning of 1930-ies between Einstein and Bohr [1, 2].

The understanding of peculiarities of the quantum world goes in many ways, one of them is creating of various "realisms", i.e. non-controversial and complete sets of properties that allow to distinct quantum and classical phenomena. Usually these properties are related to the classical world and to the classical paradigm. Usage of various of sets of properties allows to formulate statements that are correct in classical world, but may be violated in quantum world. Note that the "non-classical world" is considered not only as the world of quantum phenomena, but also as any possible phenomena beyond the quantum physics [3, 4], for which there are no experimental evidence.

First concept in the "realism" series is the concept of local realism that was formulated in the famous work of Einstein, Podolsky and Rosen [5]. In the framework of the concept of local realism the hypothesis of hidden variables has been actively discussed, and Bell inequalities $[6,7]$ has been formulated, as well as the Clauser-Horn-Shimony-Holt inequalities [8] and Wigner inequality [9]. Next "realism" - the macroscopic one - has appeared in the work of Leggett and Garg [10]. The Leggett-Garg inequalieies were obtained basing on the

\footnotetext{
*e-mail: nnikit@mail.cern.ch

**e-mail: ktoms@mail.cern.ch
} 
principle of macroscopic realism. Deeper investigation of macroscopic realism led to formulation of "No-signaling in time" condition (NSIT) [11]. NSIT requires that probabilities $w\left(q_{j}, q_{i}, \ldots \mid t_{j}, t_{i}, \ldots\right)$ of measuring of an observable $Q$ at time $t_{i}, t_{j}>t_{i}$ and so on, do not depend on the state of the observable $Q$ at time $t_{k} \neq\left\{t_{i}, t_{j}, \ldots\right\}$. Denoting $Q\left(t_{i}\right)$ as $q_{i}$, the NSIT may be written as

$$
\sum_{q_{k}} w\left(q_{j}, q_{k}, q_{i}, \ldots \mid t_{j}, t_{k}, t_{i}, \ldots\right) \equiv w\left(q_{j}, q_{i}, \ldots \mid t_{j}, t_{i}, \ldots\right) .
$$

However both local and macroscopic realisms are not well suited for experimental tests in high energy physics. Bell inequalities, obtained in the framework of local realism are static by derivation, but in high energy physics it is not possible to "switch off" interactions of quantum fields in time. Tests of the time-dependent Leggett-Garg inequalities require technique of non-invasive measurements, that is not implementable at comtemporary accelerator experiments.

Some time ago the hypothesis of realism $(\mathrm{RH})[12,13]$ has appeared in literature, which can be used for tests at high energy physics experiments. Basing on the work [14] we will consider this hypothesis compraising the following assumptions:

1) At any time $t_{i}$ a system is in a "real physical state", which exists objectively and does not depend on an observer. "Real physical state" is fully described by a set of physical variables. These variables are not supposed to be jointly measurable by any macroscopic device.

2) Observable physical states of a system are distinct because of the values of the variables that can be jointly measurable in this system at some time $t_{i}$.

I.e. under "Real physical state" we understand the ontic state $[12,15]$, which, from the observer's point of view, may appear both ontic state, and epistemic state. In order to obtain meaningful inequalities, it is necessary to expand the RH with the following requirements:

3) NSIT and/or NSC are hold for the considered system at any time $t_{i}$.

4) The observer has free will to plan, perform, and analyze the results of the experiments.

The paper is organized as follows. In the "Introduction" we give a definition of the hypothesis of realism and discuss the logic of the work. In Section 2 we give a derivation of the modified Wigner inequality for test of the RH. Section 3 contains an example of violation of the modified Wigner inequality in quantum mechanics. The "Conclusion" contains the summary of the main results.

\section{The inequality for the test of the hypothesis of realism}

Let us consider a closed physical system which consists of two subsystems, "1" and "2". Suppose that in each of the subsystems there is a dichotomic variable $Q^{(\eta)}(t)$, where $\eta=$ $\{1,2\}$ is the subsystem index. At any time $t_{i}$ both variables $Q^{(\eta)}(t)$ must have a defined value $q_{i}^{(\eta)}= \pm 1$. Let us consider three times, $t_{3}>t_{2}>t_{1}$. According to experimentalist's free will, at any two of these three times a measurement of $Q^{\eta}$ is performed. At time $t_{1}$ there is an anticorrelation between dichotomic variables $Q^{(1)}(t)$ and $Q^{(2)}(t)$ like $Q^{(1)}\left(t_{1}\right)=-Q^{(2)}\left(t_{1}\right)$, or

$$
q_{1 \pm}^{(1)}=-q_{1 \mp}^{(2)} \text {. }
$$

If at time $t_{1}$ a destructive/invasive measurement of $Q^{(\eta)}\left(t_{1}\right)$ occured, then at times $t_{2}$ and $t_{3}$ there is no correlation between $Q^{(1)}(t)$ and $Q^{(2)}(t)$. If at time $t_{1}$ there is no measurement of $Q^{(\eta)}\left(t_{1}\right)$, then the anticorrelation (2) will hold at $t_{2}$. Note, that by definition at $t_{3}$ the anticorrelation between the observables $Q^{(1)}(t)$ and $Q^{(2)}(t)$ cannot be observed under any conditions. 
Let us stress again the fact that the above situation is different to the situation with test of Legget-Garg inequality, when the NIM condition is hold. When testing the hypothesis of realism we suppose that any measurement performed on the system breaks the correlation.

We introduce a space of elementary outcomes $\omega^{(\widetilde{L G})} \in \Omega^{(\widetilde{L G})}$, which consists of the aggregates

$$
\left\{q_{3 \alpha}^{(2)}, q_{2 \beta}^{(2)}, q_{1 \gamma}^{(2)}, q_{3 \alpha^{\prime}}^{(1)}, q_{2 \beta^{\prime}}^{(1)}, q_{1 \gamma^{\prime}=-\gamma}^{(1)}\right\}
$$

where the indices $\left\{\alpha, \beta, \gamma, \alpha^{\prime}, \beta^{\prime}, \gamma^{\prime}\right\}=\{+,-\}$, and the anticorrelation condition (2) is taken

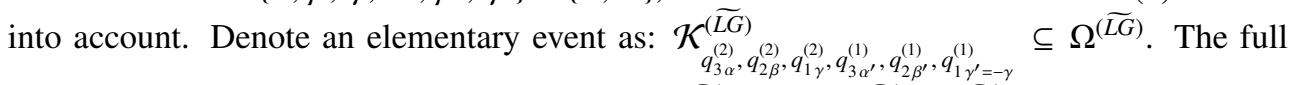
aggregate of such events forms a $\sigma$-algebra $\mathcal{F}^{(\widetilde{L G})}$. On $\left(\Omega^{(\widetilde{L G})}, \mathcal{F}^{(\widetilde{L G})}\right)$ let us introduce a non-negative $\sigma$-additive measure $w\left(\omega^{(\widetilde{L G})}, q_{3 \alpha}^{(2)}, q_{2 \beta}^{(2)}, q_{1 \gamma}^{(2)}, q_{3 \alpha^{\prime}}^{(1)}, q_{2 \beta^{\prime}}^{(1)}, q_{1 \gamma^{\prime}=-\gamma}^{(1)} \mid t_{3}, t_{2}, t_{1}\right)$. The triplet $\left(\Omega^{(\widetilde{L G})}, \mathcal{F}^{(\widetilde{L G})}, w(\ldots)\right)$ is a probabilistic model, which will be used to test the hypothesis of realism.

For rigorous application in the framework of Kolmogorov axiomatics, the mathematical form of the no-signaling in time condition (1) should be corrected for the definition of the elementary outcome as follows:

$$
\sum_{\omega_{i j . .}^{(\widetilde{L G})} \in \mathcal{K}_{i j . .}^{(\widetilde{L G})}} \sum_{q_{k}} w\left(\omega_{i j \ldots}^{(\widetilde{L G})}, q_{j}, q_{k}, q_{i}, \ldots \mid t_{j}, t_{k}, t_{i}, \ldots\right)=w\left(q_{j}, q_{i}, \ldots \mid t_{j}, t_{i}, \ldots\right)
$$

We now prove the inequality which is analogous to Wigner inequality [9]. We introduce an event:

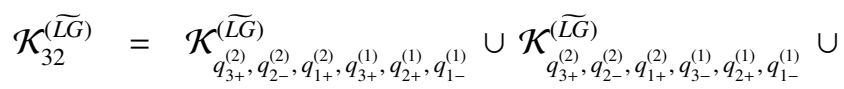

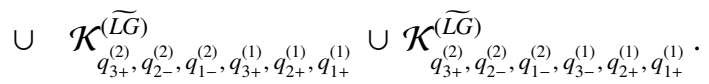

Equation (4) takes into account that the variables $Q^{(1)}(t)$ and $Q^{(2)}(t)$ are anticorrelated at time $t_{1}$, as well as at time $t_{2}$, because there has been no measurement at $t_{1}$. Then, taking into account (3), we may write:

$$
\begin{aligned}
w\left(q_{3+}^{(2)}, q_{2+}^{(1)} \mid t_{3}, t_{2}\right)= & \sum_{\substack{\omega_{32}^{(\widetilde{L G})} \in \mathcal{K}_{32}^{(\widetilde{L G})} \\
q_{3}^{(1)}}} \sum_{q_{1}^{(1)}} \sum_{q_{1}^{(2)}} \delta_{-q_{1}^{(1)}} q_{1}^{(2)} \\
& w\left(\omega_{32}^{(\widetilde{L G})}, q_{3+}^{(2)}, q_{2-}^{(2)}, q_{1}^{(2)}, q_{3}^{(1)}, q_{2+}^{(1)}, q_{1}^{(1)} \mid t_{3}, t_{2}, t_{1}\right),
\end{aligned}
$$

where $\delta_{i j}$ is the Kronecker delta. Then let us introduce another two events. These are:

$$
\begin{aligned}
& \mathcal{K}_{31}^{(\widetilde{L G})}=\mathcal{K}_{q_{3+}^{(2)}, q_{2+}^{(2)}, q_{1-}^{(2)}, q_{3+}^{(1)}, q_{2+}^{(1)}, q_{1+}^{(1)}}^{\left(\widetilde{N_{1}}\right.} \cup \mathcal{K}_{q_{3+}^{(2)}, q_{2+}^{(2)}, q_{1-}^{(2)}, q_{3+}^{(1)}, q_{2-}^{(1)}, q_{1+}^{(1)}}^{(\widetilde{L G})}
\end{aligned}
$$

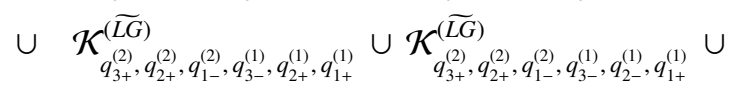

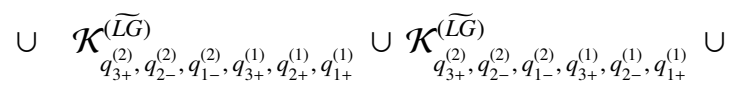

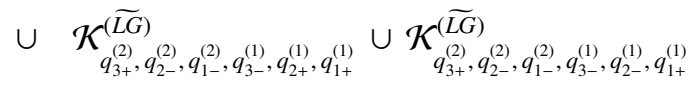


and

$$
\begin{aligned}
& \mathcal{K}_{12}^{(\widetilde{L G})}=\mathcal{K}_{q_{3+}^{(2)}}^{(\widetilde{L G})}, q_{2+}^{(2)}, q_{1+}^{(2)}, q_{3+}^{(1)}, q_{2+}^{(1)}, q_{1-}^{(1)} \cup \mathcal{K}_{q_{3+}^{(2)}, q_{2+}^{(2)}, q_{1+}^{(2)}, q_{3-}^{(1)}, q_{2+}^{(1)}, q_{1-}^{(1)}}^{(\widetilde{L G}} \cup
\end{aligned}
$$

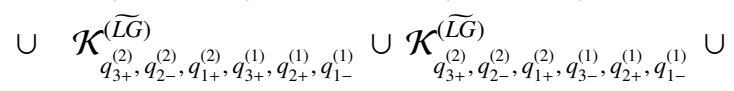

$$
\begin{aligned}
& \cup \mathcal{K}_{q_{3-}^{(2)}, q_{2+}^{(2)}, q_{1+}^{(2)}, q_{3+}^{(1)}, q_{2+}^{(1)}, q_{1-}^{(1)}}^{(\widetilde{L})} \cup \mathcal{K}_{q_{3-}^{(2)}, q_{2+}^{(2)}, q_{1+}^{(2)}, q_{3-}^{(1)}, q_{2+}^{(1)}, q_{1-}^{(1)}}^{(\bar{L})} \\
& \cup \mathcal{K}_{q_{3-}^{(2)}, q_{2-}^{(2)}, q_{1+}^{(2)}, q_{3+}^{(1)}, q_{2+}^{(1)}, q_{1-}^{(1)}}^{\left(\widetilde{L} \mathcal{K}_{q_{3-}}^{(\widetilde{L G})} q_{2-}^{(2)}, q_{1+}^{(2)}, q_{3-}^{(1)}, q_{2+}^{(1)}, q_{1-}^{(1)}\right.} \text {. }
\end{aligned}
$$

Equations (6) and (7) take into account that the anticorrelation between $Q^{(1)}(t)$ and $Q^{(2)}(t)$ exists only at the time $t_{1}$, when the first measurement of one of the observables takes place. This distinguishes (6) and (7) from (4).

For events (6) and (7) we define probabilities

$$
\begin{aligned}
w\left(q_{3+}^{(2)}, q_{1+}^{(1)} \mid t_{3}, t_{1}\right)= & \sum_{\substack{\omega_{31}^{(\widetilde{L G}} \in \mathcal{K}_{31}^{(\widetilde{L G})(1)} \\
q_{3}^{(1)}}} \sum_{q_{2}^{(1)}} \sum_{q_{2}^{(2)}} \\
& w\left(\omega_{31}^{(\widetilde{L G})}, q_{3+}^{(2)}, q_{2}^{(2)}, q_{1-}^{(2)}, q_{3}^{(1)}, q_{2}^{(1)}, q_{1+}^{(1)} \mid t_{3}, t_{2}, t_{1}\right)
\end{aligned}
$$

and

$$
\begin{aligned}
w\left(q_{1+}^{(2)}, q_{2+}^{(1)} \mid t_{2}, t_{1}\right)= & \sum_{\substack{\omega_{12}^{(\widetilde{L G})} \in \mathcal{K}_{12}^{(\widetilde{L G})} \\
q_{2}^{(2)}}} \sum_{q_{3}^{(2)}} \sum_{q_{3}^{(1)}} \\
& w\left(\omega_{12}^{(\widetilde{L G})}, q_{3}^{(2)}, q_{2}^{(2)}, q_{1+}^{(2)}, q_{3}^{(1)}, q_{2+}^{(1)}, q_{1-}^{(1)} \mid t_{3}, t_{2}, t_{1}\right) .
\end{aligned}
$$

The sums (8) and (9) are defined for the event $\mathcal{K}_{321}^{(\widetilde{L G})}=\mathcal{K}_{31}^{(\widetilde{L G})} \cup \mathcal{K}_{12}^{(\widetilde{L G})}$. This event also contains the event $\mathcal{K}_{32}^{(\widetilde{L G})}$. Taking into account the non-negativity of the probability measure from (5), (8), and (9) we find that for event $\mathcal{K}_{321}^{(\widetilde{L G})}$ the following is satisfied:

$$
w\left(q_{3+}^{(2)}, q_{2+}^{(1)} \mid t_{3}, t_{2}\right) \leq w\left(q_{3+}^{(2)}, q_{1+}^{(1)} \mid t_{3}, t_{1}\right)+w\left(q_{1+}^{(2)}, q_{2+}^{(1)} \mid t_{2}, t_{1}\right) .
$$

Inequality (10) is the main result of the current work. It is obtained using the hypothesis of realism and no-signaling in time condition. Violation of the inequality (10) in experiments with correlated systems will prove the unsoundness of the hypothesis of realism.

\section{Test of the hypothesis of realism in the neutral pseudoscalar meson systems}

At the first step we consider one of the neutral pseudoscalar mesons $M=\left\{K, D, B^{0}, B_{s}\right\}$ from the $M \bar{M}$ pair. We define an observable $Q$ as the flavor variable according to the rule: $Q=+1$ for meson flavor $M$ and $Q=-1$ for meson flavor $\bar{M}$. The state of each of the mesons in the meson pair will be described in two-dimensional Hilbert space $\mathcal{H}^{(\alpha)}$, where $\alpha=\{1,2\}$. We start our consideration from the basis of the states with a defined flavor:

$$
\left|M^{(\alpha)}\right\rangle=\left(\begin{array}{l}
1 \\
0
\end{array}\right), \quad\left|\bar{M}^{(\alpha)}\right\rangle=\left(\begin{array}{l}
0 \\
1
\end{array}\right) .
$$

Also let us define an arbitrary phase of $C P$-conjugation, that

$$
\hat{C} \hat{P}\left|M^{(\alpha)}\right\rangle=\left|\bar{M}^{(\alpha)}\right\rangle, \quad \hat{C} \hat{P}\left|\bar{M}^{(\alpha)}\right\rangle=\left|M^{(\alpha)}\right\rangle .
$$


In the basis (11) one can to define states with defined $C P$-parity

$$
\left|M_{1}^{(\alpha)}\right\rangle=\frac{1}{\sqrt{2}}\left(\left|M^{(\alpha)}\right\rangle+\left|\bar{M}^{(\alpha)}\right\rangle\right), \quad\left|M_{2}^{(\alpha)}\right\rangle=\frac{1}{\sqrt{2}}\left(\left|M^{(\alpha)}\right\rangle-\left|\bar{M}^{(\alpha)}\right\rangle\right)
$$

and with a certain values of the masses and lifetimes

$$
\left|M_{L}^{(\alpha)}\right\rangle=p\left|M^{(\alpha)}\right\rangle+q\left|\bar{M}^{(\alpha)}\right\rangle, \quad\left|M_{H}^{(\alpha)}\right\rangle=p\left|M^{(\alpha)}\right\rangle-q\left|\bar{M}^{(\alpha)}\right\rangle .
$$

$C P$-eigenstates are orthogonal, but $\left\langle M_{L}^{(\alpha)} \mid M_{H}^{(\alpha)}\right\rangle=|p|^{2}-|q|^{2} \neq 0$. Complex parameters $p$ and $q$ suit the following normalization condition:

$$
\left\langle M_{L}^{(\alpha)} \mid M_{L}^{(\alpha)}\right\rangle=\left\langle M_{H}^{(\alpha)} \mid M_{H}^{(\alpha)}\right\rangle=|p|^{2}+|q|^{2}=1 .
$$

Let $\hat{D}$ be the operator of converting to basis where the states $\left|M_{L, H}^{(\alpha)}\right\rangle$ are orthogonal. This operator has the following form:

$$
\hat{D}^{(\alpha)}=\frac{1}{2 p q}\left(\begin{array}{cc}
q & p \\
q & -p
\end{array}\right)
$$

In the basis where the states $\left|M_{L}^{(\alpha)}\right\rangle$ and $\left|M_{H}^{(\alpha)}\right\rangle$ are orthogonal, the operator of time evolution has the following form:

$$
\hat{U}^{(\alpha)}(t)=\left(\begin{array}{cc}
e^{-i E_{L} t} & 0 \\
0 & e^{-i E_{H} t}
\end{array}\right)
$$

and

$$
E_{L}=m_{L}-\frac{i}{2} \Gamma_{L} ; \quad E_{H}=m_{H}-\frac{i}{2} \Gamma_{H}
$$

are the complex energies related to the states $\left|M_{L}^{(\alpha)}\right\rangle$ and $\left|M_{H}^{(\alpha)}\right\rangle$ accordingly. For the subsequent calculations let us use the following definitions:

$$
\Delta m=m_{H}-m_{L}, \quad \Delta \Gamma=\Gamma_{H}-\Gamma_{L}, \quad \Gamma=\frac{1}{2}\left(\Gamma_{H}+\Gamma_{L}\right) .
$$

The inverse operator for $\hat{D}^{(\alpha)}$, has the following form:

$$
\left(\hat{D}^{(\alpha)}\right)^{-1}=\left(\begin{array}{cc}
p & p \\
q & -q
\end{array}\right)
$$

It changes the orthogonal states $\left|M_{L}^{(\alpha)}\right\rangle$ and $\left|M_{H}^{(\alpha)}\right\rangle$ back into non-orthogonal. Finally in the space $\mathcal{H}^{(\alpha)}$ let us introduce $S$-matrix

$$
\hat{S}^{(\alpha)}(t)=\left(\hat{D}^{(\alpha)}\right)^{-1} \hat{U}^{(\alpha)}(t) \hat{D}^{(\alpha)} .
$$

This matrix satisfies the following group property:

$$
\hat{S}^{(\alpha)}\left(t_{1}+t_{2}\right)=\hat{S}^{(\alpha)}\left(t_{1}\right) \hat{S}^{(\alpha)}\left(t_{2}\right),
$$

as the evolution matrix $\hat{U}^{(\alpha)}(t)$ satisfies it.

Using the above technique it is easy to calculate any time evolutions of any states of pseudoscalar mesons. For example:

$$
\left\{\begin{array}{l}
\left|M^{(\alpha)}(t)\right\rangle=\hat{S}^{(\alpha)}(t)\left|M^{(\alpha)}\right\rangle=g_{+}(t)\left|M^{(\alpha)}\right\rangle-\frac{q}{p} g_{-}(t)\left|\bar{M}^{(\alpha)}\right\rangle \\
\left|\bar{M}^{(\alpha)}(t)\right\rangle=\hat{S}^{(\alpha)}(t)\left|\bar{M}^{(\alpha)}\right\rangle=g_{+}(t)\left|\bar{M}^{(\alpha)}\right\rangle-\frac{p}{q} g_{-}(t)\left|M^{(\alpha)}\right\rangle
\end{array},\right.
$$


where $g_{ \pm}(t)=\frac{1}{2}\left(e^{-i E_{H} t} \pm e^{-i E_{L} t}\right)$. Functions $g_{ \pm}(t)$ satisfy the following conditions:

$$
\begin{aligned}
& \left|g_{ \pm}(t)\right|^{2}=\frac{e^{-\Gamma t}}{2}\left(\operatorname{ch}\left(\frac{\Delta \Gamma t}{2}\right) \pm \cos (\Delta m t)\right), \\
& g_{+}^{*}(t) g_{-}(t)=-\frac{e^{-\Gamma t}}{2}\left(\operatorname{sh}\left(\frac{\Delta \Gamma t}{2}\right)+i \sin (\Delta m t)\right) .
\end{aligned}
$$

Also from the group property (17) it follows that:

$$
\begin{aligned}
& g_{+}\left(t_{2}+t_{1}\right)=g_{+}\left(t_{2}\right) g_{+}\left(t_{1}\right)+g_{-}\left(t_{2}\right) g_{-}\left(t_{1}\right), \\
& g_{-}\left(t_{2}+t_{1}\right)=g_{+}\left(t_{2}\right) g_{-}\left(t_{1}\right)+g_{-}\left(t_{2}\right) g_{+}\left(t_{1}\right) .
\end{aligned}
$$

At the second step let us consider a pair of neutral pseudoscalar mesons that at time $t_{1}=0$ exists in a flavour Bell-entangled state:

$$
\left|\Psi^{+}\right\rangle=\frac{1}{\sqrt{2}}\left(\left|M^{(2)}\right\rangle \otimes\left|\bar{M}^{(1)}\right\rangle+\left|\bar{M}^{(2)}\right\rangle \otimes\left|M^{(1)}\right\rangle\right) .
$$

Evolution of the state $\left|\Psi^{+}\right\rangle$is described in the Hilbert space $\mathcal{H}=\mathcal{H}^{(1)} \otimes \mathcal{H}^{(2)}$. In this space the $S$-matrix has the form:

$$
\hat{S}(t)=\hat{S}^{(1)}(t) \otimes \hat{S}^{(2)}(t),
$$

and projectors to states $\left|M^{(1)}\right\rangle$ and $\left|M^{(2)}\right\rangle$ :

$$
\hat{\mathcal{P}}_{M}^{(1)}=\hat{P}_{M}^{(1)} \otimes \hat{1}^{(2)}, \quad \hat{\mathcal{P}}_{M}^{(2)}=\hat{1}^{(1)} \otimes \hat{P}_{M}^{(2)},
$$

where $\hat{1}^{(\alpha)}-$ is a unitary operator in the space $\mathcal{H}^{(\alpha)}$ and $\hat{P}_{M}^{(\alpha)}=\left|M^{(\alpha)}\right\rangle\left\langle M^{(\alpha)}\right|-$ is a projector to the state $\left|M^{(\alpha)}\right\rangle$ in the space $\mathcal{H}^{(\alpha)}$.

Let in the time $t_{1}=0$ our system to be in the state $\left|\Psi^{+}\right\rangle$, then at the time $t_{2}>t_{1}$ the first meson to be measured as the state " $M$ ", and at the moment of time $t_{3}>t_{2}$ the second meson to be also measured as the state " $M$ ". Then at time $t_{3}$ the pair of pseudoscalar mesons is in the state:

$$
\begin{aligned}
\left|\Psi\left(t_{3}, t_{2},\left[t_{1}\right]\right)\right\rangle & =\hat{\mathcal{P}}_{M}^{(2)} \hat{S}\left(t_{3}-t_{2}\right) \hat{\mathcal{P}}_{M}^{(1)} \hat{S}\left(t_{2}-t_{1}\right)\left|\Psi^{+}\left(t_{1}\right)\right\rangle= \\
& =-\frac{1}{\sqrt{2}} \frac{p}{q} g_{-}\left(t_{2}+t_{3}\right)\left(g_{+}\left(\Delta t_{32}\right)\left|M^{(1)}\right\rangle-\frac{q}{p} g_{-}\left(\Delta t_{32}\right)\left|\bar{M}^{(1)}\right\rangle\right) \otimes\left|M^{(2)}\right\rangle
\end{aligned}
$$

where $\Delta t_{32}=t_{3}-t_{2}$ and $\left[t_{i}\right]$ defines the time when there were no measurements. Using the relation for the state vector (19), the probability $w\left(q_{3+}^{(2)}, q_{2+}^{(1)} \mid t_{3}, t_{2}\right)$ may be written as:

$$
\begin{aligned}
w\left(q_{3+}^{(2)}, q_{2+}^{(1)} \mid t_{3}, t_{2}\right)= & \frac{1}{4} e^{-2 \Gamma t_{3}} \operatorname{ch}\left(\frac{\Delta \Gamma \Delta t_{32}}{2}\right) \\
& {\left[\operatorname{ch}\left(\frac{\Delta \Gamma\left(t_{2}+t_{3}\right)}{2}\right)-\cos \left(\Delta m\left(t_{2}+t_{3}\right)\right)\right] . }
\end{aligned}
$$

In order to introduce an example of violation of (10) let us write two more state vectors. First is related to the fact that at the time $t_{1}=0$ the pair of pseudoscalar mesons was in the state $\left|\Psi^{+}\right\rangle$. Then at $t_{1}$ the first meson was measured as the state " $M$ ", and then at $t_{3}>t_{1}$ the second meson also was measured as the state " $M$ ". At the time $t_{2}$ measurement is not performed. $t_{3}>t_{2}>t_{1}$. Then:

$$
\begin{aligned}
\left|\Psi\left(t_{3},\left[t_{2}\right], t_{1}\right)\right\rangle & =\hat{\mathcal{P}}_{M}^{(2)} \hat{S}\left(t_{3}-t_{2}\right) \hat{S}\left(t_{2}-t_{1}\right) \hat{\mathcal{P}}_{M}^{(1)}\left|\Psi^{+}\left(t_{1}\right)\right\rangle= \\
& =\hat{\mathcal{P}}_{M}^{(2)} \hat{S}\left(t_{3}-t_{1}\right) \hat{\mathcal{P}}_{M}^{(1)}\left|\Psi^{+}\left(t_{1}\right)\right\rangle= \\
& =-\frac{1}{\sqrt{2}} \frac{p}{q} g_{-}\left(t_{3}\right)\left(g_{+}\left(t_{3}\right)\left|M^{(1)}\right\rangle-\frac{q}{p} g_{-}\left(t_{3}\right)\left|\bar{M}^{(1)}\right\rangle\right) \otimes\left|M^{(2)}\right\rangle .
\end{aligned}
$$


The second state vector is related to the fact that at $t_{1}=0$ the system was in the state $\left|\Psi^{+}\right\rangle$. At the same time the second meson is measured as the state " $M$ ". At $t_{2}>t_{1}$ the first meson is measured in the state " $M$ ". It is necessary to find a state vector at $t_{3}>t_{2}$, where no measurements take place. Have:

$$
\begin{aligned}
\left|\Psi\left(\left[t_{3}\right], t_{2}, t_{1}\right)\right\rangle & =\hat{S}\left(t_{3}-t_{2}\right) \hat{\mathcal{P}}_{M}^{(1)} \hat{S}\left(t_{2}-t_{1}\right) \hat{\mathcal{P}}_{M}^{(2)}\left|\Psi^{+}\left(t_{1}\right)\right\rangle= \\
& =-\frac{1}{\sqrt{2}} \frac{p}{q} g_{-}\left(t_{2}\right)\left(g_{+}\left(\Delta t_{32}\right)\left|M^{(1)}\right\rangle-\frac{q}{p} g_{-}\left(\Delta t_{32}\right)\left|\bar{M}^{(1)}\right\rangle\right) \otimes \\
& \otimes\left(g_{+}\left(t_{3}\right)\left|M^{(2)}\right\rangle-\frac{q}{p} g_{-}\left(t_{3}\right)\left|\bar{M}^{(2)}\right\rangle\right)
\end{aligned}
$$

Using (21) and (22) we obtain:

$$
w\left(q_{3+}^{(2)}, q_{1+}^{(1)} \mid t_{3}, t_{1}\right)=\frac{1}{4} e^{-2 \Gamma t_{3}} \operatorname{ch}\left(\frac{\Delta \Gamma \Delta t_{3}}{2}\right)\left[\operatorname{ch}\left(\frac{\Delta \Gamma t_{3}}{2}\right)-\cos \left(\Delta m t_{3}\right)\right]
$$

and

$$
\begin{aligned}
w\left(q_{1+}^{(2)}, q_{2+}^{(1)} \mid t_{2}, t_{1}\right)= & \frac{1}{4} e^{-2 \Gamma t_{3}} \operatorname{ch}\left(\frac{\Delta \Gamma \Delta t_{32}}{2}\right) \operatorname{ch}\left(\frac{\Delta \Gamma t_{3}}{2}\right) \\
& {\left[\operatorname{ch}\left(\frac{\Delta \Gamma t_{2}}{2}\right)-\cos \left(\Delta m t_{2}\right)\right] . }
\end{aligned}
$$

Denote

$$
\kappa=\frac{\Delta \Gamma}{2 \Delta m}, \quad \alpha=\Delta m t_{3}, \quad \beta=\Delta m t_{2} .
$$

Then substituting (20), (23), (24) and (25) into (10) leads to the following inequality:

$$
\begin{aligned}
& {[\operatorname{ch}(\kappa(\alpha+\beta))-\cos (\alpha+\beta)] \operatorname{ch}(\kappa(\alpha-\beta)) \leq } \\
\leq & {[\operatorname{ch}(\kappa \alpha)-\cos (\alpha)] \operatorname{ch}(\kappa \alpha)+[\operatorname{ch}(\kappa \beta)-\cos (\beta)] \operatorname{ch}(\kappa(\alpha-\beta)) \operatorname{ch}(\kappa \alpha) . }
\end{aligned}
$$

In order to simplify the above inequality let us consider $B_{s} \bar{B}_{s}-$ meson pairs. For $B_{s}-$ meson $\Delta \Gamma \approx-6.0 \times 10^{-11} \mathrm{MeV}$ and $\Delta m \approx 1.2 \times 10^{-8} \mathrm{MeV}$ [16]. Hence $\kappa \approx-2.5 \times 10^{-3}$. I.e. violation of (26) may be considered in $\kappa=0$ regime. In this case inequality (26) turns into simple relation:

$$
\cos (\alpha)+\cos (\beta)-\cos (\alpha+\beta) \leq 1
$$

for $\alpha>\beta>0$. Choose $\alpha=\frac{3 \pi}{8}$ and $\beta=\frac{3 \pi}{10}$. Then $\cos \alpha \approx=0.383, \cos \beta \approx 0.588$, and $\cos (\alpha+\beta) \approx-0.522$, which leads to violation of inequality (27), and consequently to violation of inequality (10). So we have shown that inequality(10) may be violated in quantum theory.

\section{Conclusion}

In the present work we have obtained a modified Wigner inequality (10) for test of the hypothesis of realism. We stressed the fact that in order to obtain the inequality (10) it is necessary to use the NSIT condition. We have shown that the inequality (10) is violated in quantum mechanics.

The authors would like to thank Prof. S. Seidel (University of New Mexico, USA) and C. Aleister (Saint Genis-Pouilly, France). The work was supported by grant 16-12-10280 of the Russian Science Foundation. One of the authors (N. Nikitin) expresses his gratitude for this support. 


\section{References}

[1] N. Bohr, Discussion with Einstein on Epistemological Problems in Atomic Physisc, in A. Einstein, philosopher-scientist (Evanston, 1949) pp. 201-241.

[2] W. Heisenberg, Physik und Philosophie (S. Hirzel Verlag, Stuttgart, 1959)

[3] S. Popescu, D. Rohrlich, Found. Phys. 24, pp.379-385 (1994).

[4] M. Pawlowski, T. Paterek, D. Kaszlikowski, V. Scarani, A. Winter and M. Zukowski, Nature 461, pp.1101-1104 (2009).

[5] A. Einstein, B. Podolsky and N. Rosen, Phys. Rev. 47, p.777 (1935).

[6] Bell J. S., Physics 1964. 1, p.195 (1964).

[7] Bell J. S., Rev. Mod. Phys. 38, p.447 (1966).

[8] Clauser J. F., Horne M. A., Shimony A., Holt R. A., Phys. Rev. Lett. 23, p.880 (1969).

[9] E. P. Wigner, Am. J. Phys. 38, 1005 (1970).

[10] A. J. Leggett and A. Garg, Phys. Rev. Lett. 54, p.857 (1985).

[11] J. Kofler and C. Brukner, Phys. Rev. A 87, 052115 (2013).

[12] M.F. Pusey, J. Barrett, T. Rudolph, Nature Phys. 8, p.476 (2012).

[13] J. A. Formaggio, D. I. Kaiser, M. M. Murskyj and T. E. Weiss, Phys. Rev. Lett. 117, 050402 (2016).

[14] N. Nikitin, K. Toms, arXiv:1808.03119 [quant-ph] (2018).

[15] N. Harrigan and R.W. Spekkens, Found. Phys. 40, p.125 (2010).

[16] N. Nikitin, V. Sotnikov and K. Toms, Phys. Rev. D 92, 016008 (2015). 